\title{
SPIHT for Generalized Multiple Description Coding
}

\author{
Agnieszka C. Miguel ${ }^{1}$ \\ Alexander E. Mohr ${ }^{2} \quad$ Eve A. Riskin ${ }^{1}$ \\ Electrical Engineering ${ }^{1}$ \\ University of Washington \\ Seattle, WA 98195-2500 \\ Computer Science and Engineering ${ }^{2}$ \\ University of Washington \\ Seattle, WA 98195-2350 \\ \{amiguel,riskin\}@isdl.ee.washington.edu \\ amohr@cs.washington.edu
}

\begin{abstract}
We present a simple and efficient scheme for using the Set Partitioning in Hierarchical Trees (SPIHT) image compression algorithm [1] in a generalized multiple description framework. To combat packet loss, controlled amounts of redundancy are added to the original data during the compression process. Unequal loss protection is implemented by varying the amount of redundancy with the importance of data. The algorithm achieves graceful degradation of image quality in the presence of increasing description loss; high image quality is obtained even when over half of the descriptions are lost.
\end{abstract}

\section{Introduction}

Most of the networks used today are based on an exchange of packets of data. When congestion occurs because of data moving from a higher to lower capacity link, the network is often forced to drop some of the packets. When there is no feedback channel that could lead to retransmission of lost packets, the decoder must recover meaningful information from the received packets alone. Packet erasure networks are often modeled through generalized multiple description coding (MDC) in which the encoder produces multiple descriptions of the source data, each exactly filling one packet [2]. Recently, the generalized MDC framework has received considerable attention and many new algorithms have been proposed $[2,3,4,5]$.

In this work, we concentrate on networks which do not provide preferential treatment to packets and instead erase them randomly. Usually, however, data vary in importance. High magnitude, low frequency data that let us recognize coarse structures in an image are much more important than low magnitude, high frequency details. For example, in a picture of a landscape, data that let us recognize mountains and forests

This work was supported by U. S. Army Research Office Grant DAAH004-96-1-0255. are more important than leaves of a tree. If the network is unable to transmit all of the data, then we would like it to discard the least important data, while retaining the most important data. We have developed an algorithm that adds controlled amounts of redundancy to the original data during the compression process to protect more important data more heavily than less important data. This method (MD-SPIHT) extends SPIHT to the generalized MDC framework.

We use SPIHT [1] because it allows for convenient determination of the importance of each data byte. By progressively ordering the data, SPIHT sends the globally most relevant information first. It produces an embedded bit stream such that its later bits refine earlier bits. Therefore, the data coded earlier are more important to the image quality than the later data. SPIHT provides high performance and low complexity but is very susceptible to packet loss. A loss in the bit stream is likely to render all following data useless. Our MD-SPIHT algorithm adapts SPIHT to lossy packet network and multiple description coding scenarios.

\section{Coding Algorithm}

To improve the robustness of SPIHT, we modify it based on the Packetized Zerotree Wavelet compression scheme (PZW) of Rogers and Cosman [6]. We eliminate the dependency between descriptions by deinterleaving the SPIHT bit stream. Spatially disperse wavelet coefficient trees are grouped together, coded with the SPIHT algorithm, and transmitted as one description. The number of trees per description can vary: the encoder examines the trees beforehand and attempts to pack as many trees as possible into one description. This method requires sending side information stating the spatial location of the first tree and the number of trees in each description. We divide the total bit rate of the image equally between all descriptions and use arithmetic coding within each description.

In this work, redundancy consists of repeated ze- 


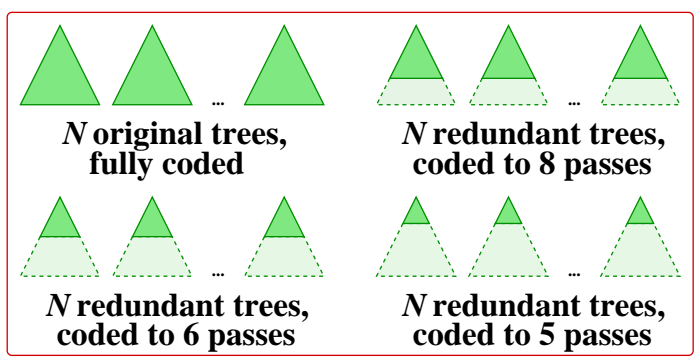

Figure 1: Example of tree selection and their coding rates in one description.

rotrees. Extra copies of each wavelet coefficient tree are placed in other descriptions. To vary the amount of redundancy according to the importance of data, we code each copy of a tree at successively lower bit rates. Each description has a variable number of fully coded original trees and $K$ sets of partially coded redundant trees corresponding to different spatial locations in the image. For example, when $K=3$, the first set of redundant trees can be coded to 8 SPIHT sorting and refinement passes, the second to 6 passes, and the third to 5 passes (see Fig. 1). Because coding to a lower pass corresponds to sending only the most significant data, the more important parts of each tree are sent in more descriptions than the less important parts. In this way, we are varying the amount of redundancy according to the importance of data.

Information about one tree is thus located in many different descriptions. As shown in the coding example in Fig. 2, if some descriptions are erased, the most important parts of the original trees in those descriptions will be recovered because their copies at lower bit rates will be present in the received descriptions. If the packet loss rate is high enough, however, some trees may be unrecoverable. In those cases we interpolate the lowest frequency coefficients from surrounding coefficients, similar to the method in PZW [6].

\section{Optimization of the Redundancy Allocation}

As in other unequal loss protection schemes [4, 5], we use an algorithm [7] to optimize the amounts of redundancy assigned to each wavelet coefficient tree in MD-SPIHT. The allocation algorithm is based on ratedistortion tradeoffs; for a given bit rate, it minimizes the expected distortion of the received data subject to a description loss model. We assume that the network behavior can be modeled by an estimator that outputs a probability mass function indicating the likelihood
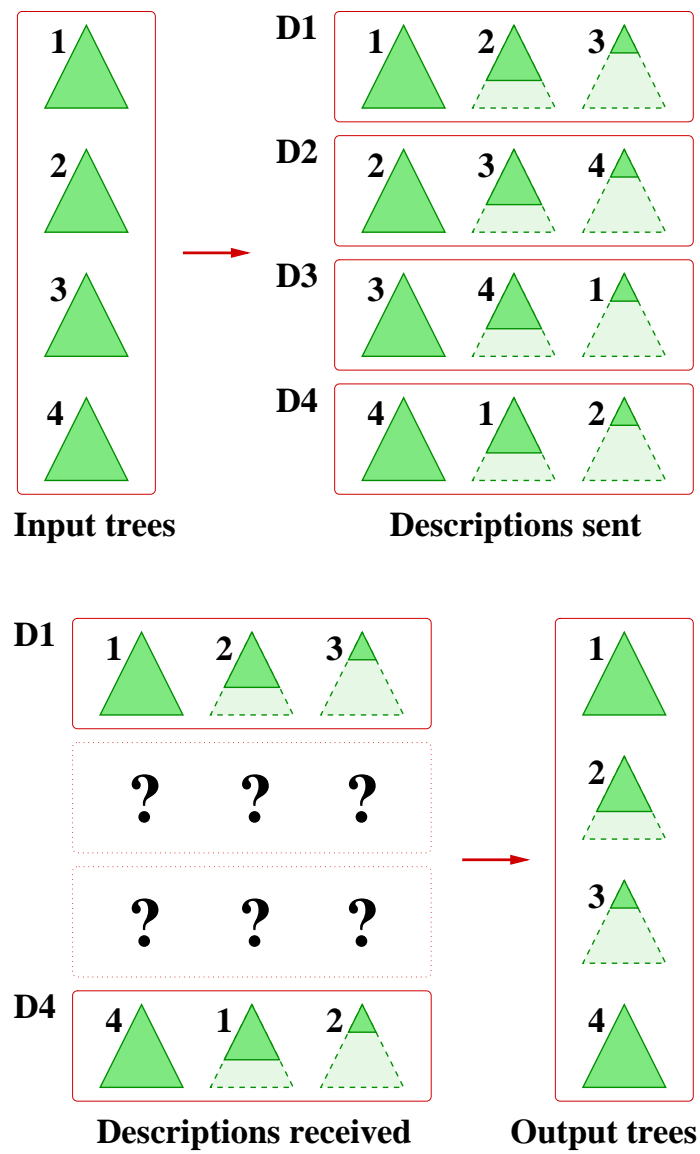

Figure 2: Coding example: four original trees are sent in four descriptions (D1-D4) with two sets of redundant trees. If two descriptions are lost (D2 and D3), the second and third trees are partially recovered from their copies in the received descriptions.

that $n$ descriptions are lost, where $n=0, \ldots, N$ and $N$ is the total number of descriptions. In addition, we assume that the original trees and their redundant copies have been already assigned to different descriptions. In this work, we optimize only the bit rates assigned to each tree and leave the optimization of the packetization algorithm for future research.

In the original SPIHT algorithm, distortion of an image can be expressed as a sum of the distortions due to decoding each of the $\mathrm{M}$ individual wavelet coefficient trees:

$$
D=\sum_{i=1}^{M} D_{i} .
$$

To incorporate the effect of expected description loss 


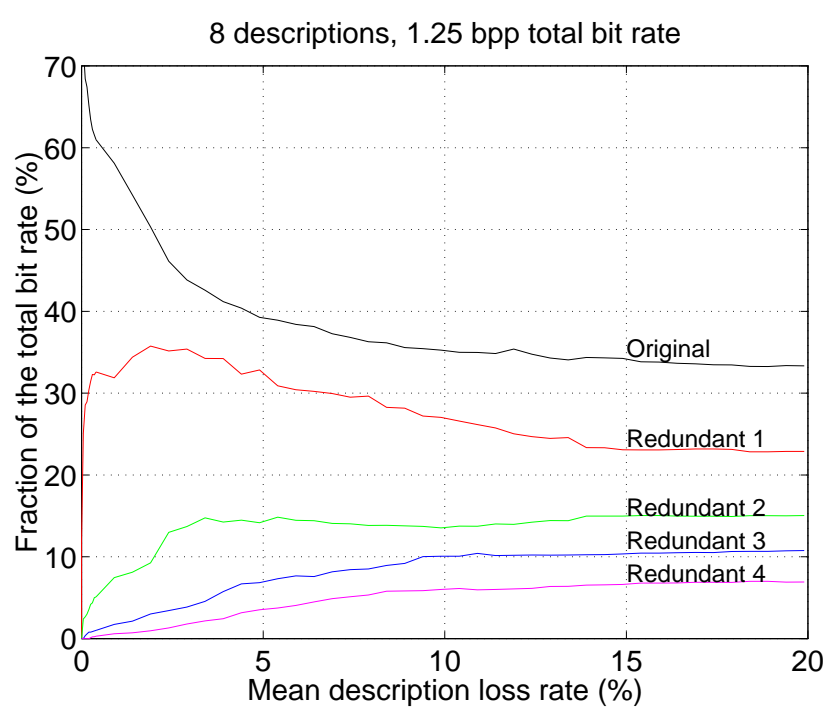

Figure 3: Contribution of the original data and 4 levels of redundant data to the total bit rate as a function of the mean expected description loss rate.

in MD-SPIHT, we expand this expression to

$$
D=\sum_{i=1}^{M} \sum_{j=0}^{N-1} p_{i j} D_{i j},
$$

where $p_{i j}$ is the probability of using the $j$ th copy of a tree $i$ and $D_{i j}$ is its distortion. The probabilities $p_{i j}$ can be easily determined from the description loss model and the packetization information.

We use the above expression to determine optimal bit rate assignments for each tree. The goal of the optimization algorithm is to minimize $D$ subject to constraints on description bit rates. We use the extended BFOS algorithm [8], which allocates bits among various tree copies such that all allocations lie on the lower convex hull of the rate-distortion curve. As expected, when the mean loss rate increases, higher bit rates are allocated to the redundant copies of each tree (Fig. 3).

The redundancy allocation algorithm outputs the number of copies of each tree and the bit rates of all redundant trees. To minimize the side information, the available bit rates of redundant trees are restricted to the end points of SPIHT sorting and refinement passes. The number of tree copies, however, can vary with each tree.

\section{Results}

We demonstrate our results on the standard $512 \times 512$ gray-scale Lena image and use a 5-level DWT decom-

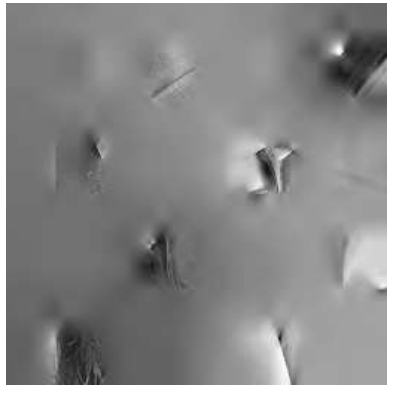

(a) PSNR $=15.77 \mathrm{~dB}$

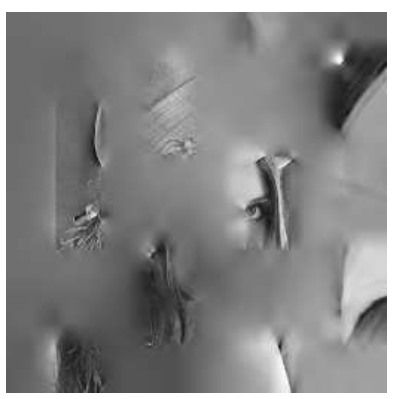

(c) $\mathrm{PSNR}=16.61 \mathrm{~dB}$

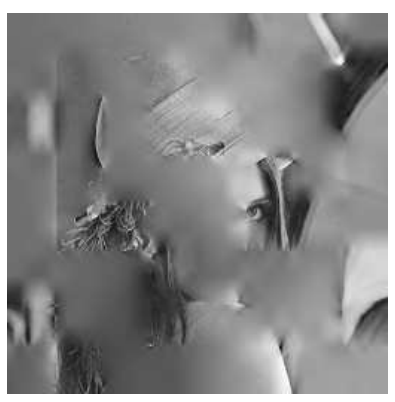

(e) $P S N R=17.55 \mathrm{~dB}$

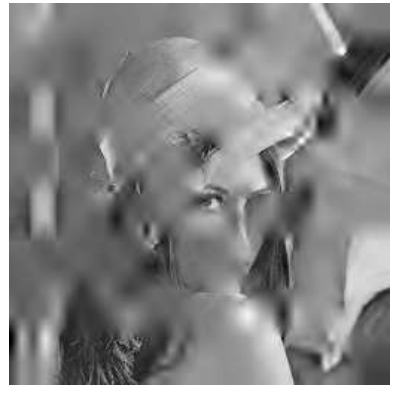

(b) PSNR $=19.15 \mathrm{~dB}$

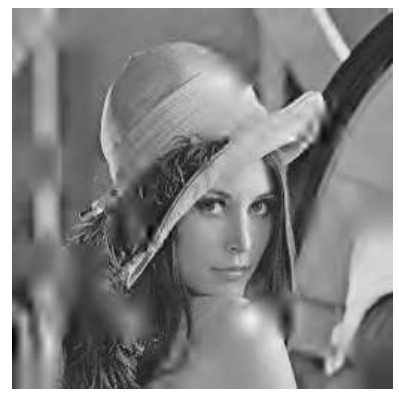

(d) $P S N R=24.43 \mathrm{~dB}$

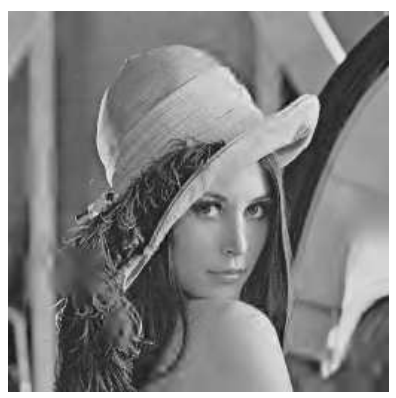

(f) PSNR $=30.63 \mathrm{~dB}$
Figure 4: Comparison of image quality for no redundancy (left column) and $35 \%$ redundancy (right col$\mathrm{umn})$. The number of received descriptions is: $(\mathrm{a}-\mathrm{b}) 1$, (c-d) 2 , and (e-f) 3 out of 8 . All results are at $1.25 \mathrm{bpp}$.

position to obtain a total of 64 trees. First, we set the number of descriptions to 8 and the total bit rate to 1.25 bpp. Fig. 4 shows images coded with MD-SPIHT for $0 \%$ and $35 \%$ redundancy. We assume that 1,2 , and 3 descriptions were received out of the 8 total. The images decoded from the redundant bit stream have superior quality and prove that adding redundancy to 


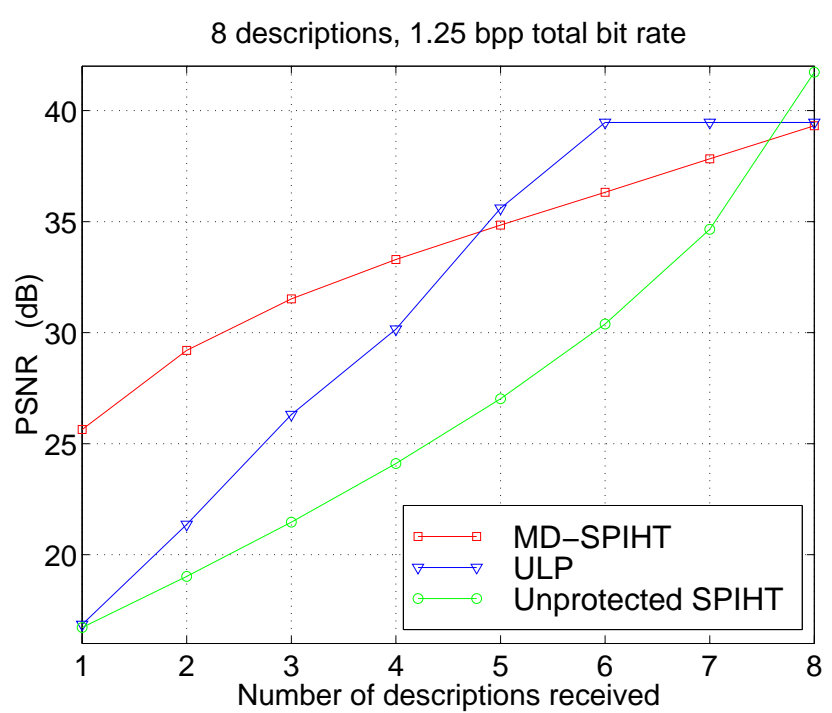

Figure 5: PSNR results for MD-SPIHT, unprotected SPIHT and the Unequal Loss Protection of Mohr et al. [5].

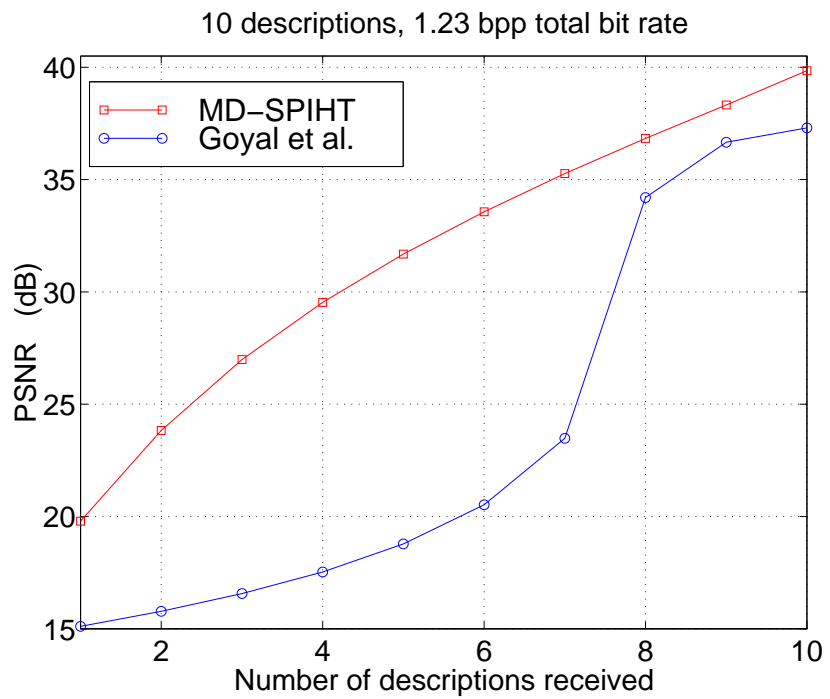

Figure 6: PSNR results for MD-SPIHT and the method of Goyal et al. [2].

the original data during the compression process is an effective way to combat description loss. In addition, the image quality in MD-SPIHT improves progressively with each description received. Since the information about one wavelet coefficient tree is included in many different descriptions, its reconstruction quality is updated whenever a higher bit rate copy is received (see Fig. 4(b), 4(d) and 4(f)).

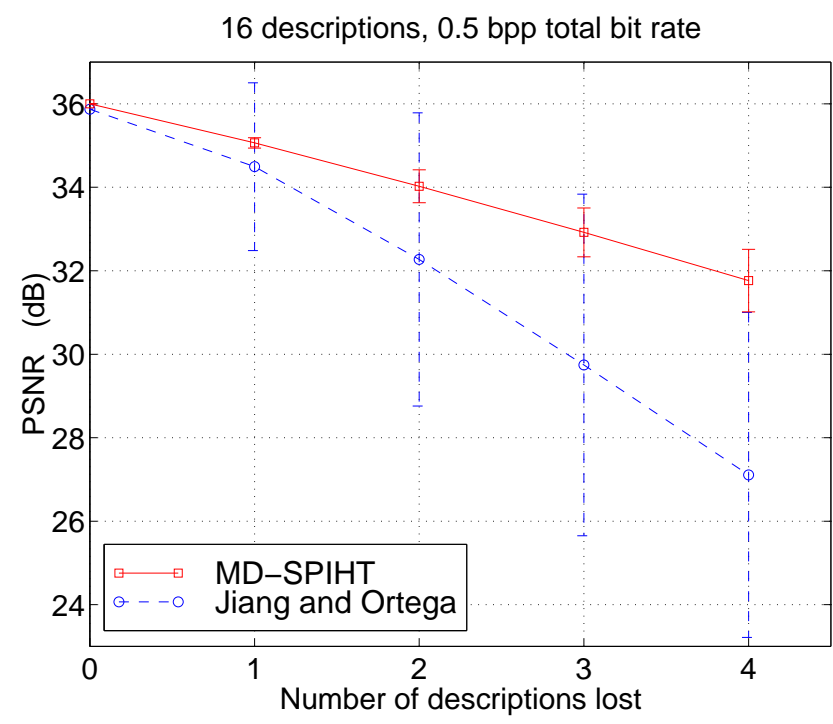

Figure 7: PSNR results for MD-SPIHT and the method of Jiang and Ortega [4]. The error bars for MD-SPIHT are shown in solid vertical lines.

We set the redundancy to $35 \%$ and compare MDSPIHT with unprotected SPIHT in which the output of the encoder is used to fill the first description, then the second description, and so on. The PSNR results for different losses are shown in Fig. 5. Our scheme clearly surpasses unprotected SPIHT if any packets are lost, but our quality is lower when there is no loss. This is expected because our effective bit rate is $0.8 \mathrm{bpp}$. We also compare the proposed method to the MDC scheme of Mohr et al. [5] that uses explicit forward error correction and sets the redundancy to $35 \%$ of the total bit rate of 1.25 bpp. Our scheme yields comparable results at high losses but is somewhat worse at losses of 3 packets or less.

Goyal et al. [2] proposed a MDC scheme that uses JPEG image compression, an 8 to 10 frame expansion, and 10 descriptions. Their total bit rate is about 1.23 bpp with $25 \%$ redundancy. As can be seen in Fig. 6, our algorithm gives more graceful degradation of image quality with increasing losses. However, much of the discrepancy between these results is due to the choice of source coding algorithms.

Jiang and Ortega [4] designed a MDC system based on polyphase transform and selective quantization. For each description, the polyphase transform is used to divide the data into original and redundant parts. Then the original data are finely quantized while the redundant data are coarsely quantized. As can be seen in Fig. 7, for the case of 16 descriptions, $0.5 \mathrm{bpp}$ total bit rate, and $20 \%$ redundancy, our algorithm gives PSNR 


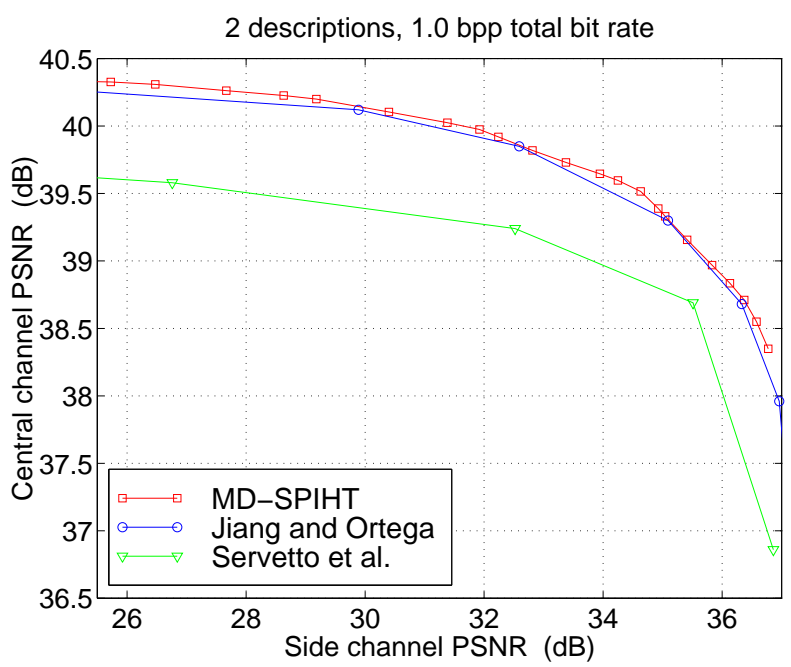

Figure 8: PSNR results for MD-SPIHT, and the methods of Jiang and Ortega [4] and Servetto et al. [9].

values similar to Jiang and Ortega's results at low losses but much higher at higher losses. The standard deviation is also much smaller in MD-SPIHT.

Next, we set the number of descriptions produced by MD-SPIHT to two and compare our method with the schemes of Servetto et al. [9] and Jiang and Ortega [4]. Clearly, our results surpass those of Servetto et al. and are comparable to Jiang and Ortega's (Fig. 8).

\section{Conclusions}

We have shown a simple and effective algorithm for adding redundancy to packetized SPIHT in a generalized multiple description framework. Based on the importance of image data, varied redundancy is added during the compression process. Results show that this method achieves graceful degradation of image quality in the presence of increasing description loss. High image quality is obtained even when over half of the descriptions are lost.

\section{Future Work}

When multiple trees from the same spatial location are received, only the tree with the highest bit rate is used for image reconstruction. We are currently trying to improve this approach by using an overcomplete frame expansion as suggested by Goyal et al. [2]. When an appropriate frame expansion is used, the additional trees will contribute useful information and improve the PSNR at low losses. We will use Chou et al.'s method for reconstructing a signal from a subset of frame coefficients based on projections onto convex sets [3].

In future research we will optimize the tree packetization, total number of descriptions, and DWT levels. In addition, the basic idea behind MD-SPIHT can be extended to other image and video compression schemes. For more information see [10].

The authors would like to thank Professor William Pearlman for the SPIHT source code and Vivek Goyal and Wenqing Jiang for providing performance results.

\section{References}

[1] A. Said and W. A. Pearlman. A new, fast, and efficient image codec based on set partitioning in hierarchical trees. IEEE Transactions on Circuits and Systems for Video Technology, 6(3):243-250, June 1996.

[2] V. K. Goyal, J. Kovačević, R. Arean, and M. Vetterli. Multiple description transform coding of images. In Proceedings of ICIP, October 1998.

[3] P. A. Chou, S. Mehrotra, and A. Wang. Multiple description decoding of overcomplete expansions using projections onto convex sets. In Proceedings Data Compression Conference, March 1999.

[4] W. Jiang and A. Ortega. Multiple description coding via polyphase transform and selective quantization. In Proceedings of SPIE: Visual Communications and Image Processing, January 1999.

[5] A. E. Mohr, E. A. Riskin, and R. Ladner. Generalized multiple description coding through unequal forward error correction. In Proceedings of ICIP, 1999.

[6] J. K. Rogers and P. C. Cosman. Robust wavelet zerotree image compression with fixed-length packetization. In Proceedings Data Compression Conference, pages 418-427, March-April 1998.

[7] A. E. Mohr, E. A. Riskin, and R. Ladner. Bit allocation for wavelet image compression and uniform bit loss. In Proceedings of CISS, March 1998.

[8] E. A. Riskin. Optimum bit allocation via the generalized BFOS algorithm. IEEE Transactions on Information Theory, 37:400-402, March 1991.

[9] S. D. Servetto, K. Ramchandran, V. Vaishampayan, and K. Nahrstedt. Multiple-description wavelet based image coding. In Proceedings of ICIP, October 1998.

[10] http://rcs.ee.washington.edu/dcl/amiguel/. 